Document downloaded from:

http://hdl.handle.net/10251/143003

This paper must be cited as:

De Luis-Fernández, B.; Llopis-Lorente, A.; Rincón, P.; Gadea Vacas, J.; Sancenón Galarza, F.; Aznar, E.; Villalonga, R.... (14-1). An Interactive Model of Communication between Abiotic Nanodevices and Microorganisms. Angewandte Chemie International Edition. 58(42):14986-14990. https://doi.org/10.1002/anie.201908867

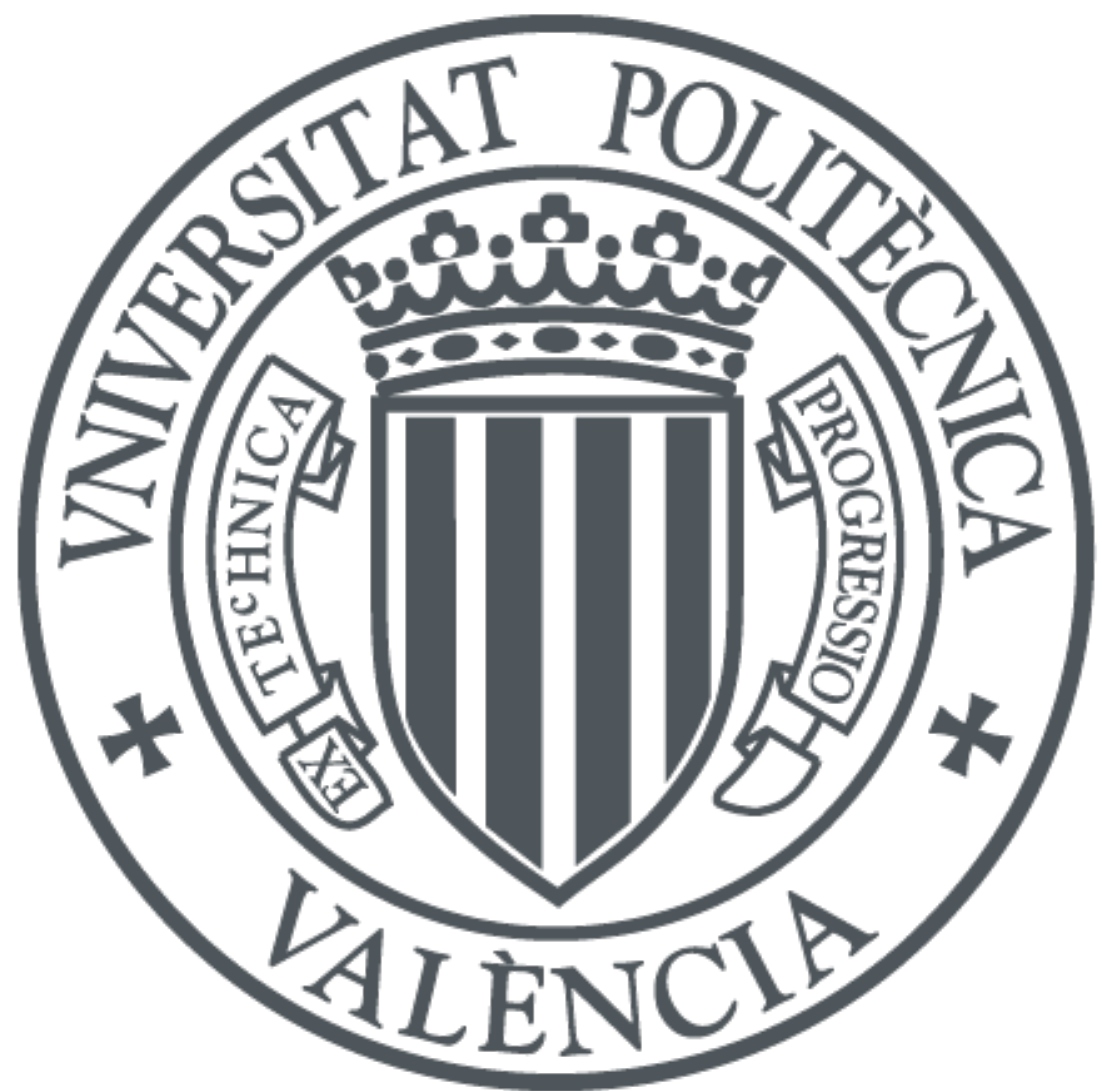

The final publication is available at

https://doi.org/10.1002/anie.201908867

Copyright John Wiley \& Sons

Additional Information

"This is the peer reviewed version of the following article: Luis, B., Llopis-Lorente, A., Rincón, P., Gadea, J., Sancenón, F., Aznar, E., Villalonga, R., Murguía, J. R., \& MartínezMáñez, R. (2019). An Interactive Model of Communication between Abiotic Nanodevices and Microorganisms. Angewandte Chemie International Edition, 58(42), 1498614990. https://doi.org/10.1002/anie.201908867, which has been published in final form at https://doi.org/10.1002/anie.201908867. This article may be used for non-commercial purposes in accordance with Wiley Terms and Conditions for Self-Archiving." 


\title{
An Interactive Model of Communication between Abiotic Nanodevices and Living Microorganisms
}

\author{
Beatriz de Luis, ${ }^{[a, b]}$ Antoni Llopis-Lorente, ${ }^{[a, b]}$ Paola Rincón, ${ }^{[a]}$ José Gadea, ${ }^{[e]}$ Félix Sancenón, ${ }^{[a, b]}$ Elena \\ Aznar, ${ }^{[a, b]}$ Reynaldo Villalonga, $\left.{ }^{[f]}\right]$ José Ramón Murguía ${ }^{[a, b]}$ and Ramón Martínez-Máñez ${ }^{*[a, b, c, d]}$
}

\begin{abstract}
The construction of communication models at the micro/nanoscale involving abiotic nanodevices and living organisms has the potential to open a wide range of applications in biomedical and communications technologies. However, this area remains almost unexplored. Here we report, as a proof of concept, a stimuliresponsive interactive paradigm of communication between yeasts (as a model microorganism) and enzyme-controlled Janus Aumesoporous silica nanoparticles. In the presence of the stimulus, the information flows from the microorganism to the nanodevice, and then returns from the nanodevice to the microorganism as a feedback.
\end{abstract}

Chemical or molecular communication based on the exchange of information by means of chemical messengers is the way of communication used by nature. For instance, neurons communicate by exchanging neurotransmitters; physiological processes are regulated by hormone molecules segregated by distant cells; and insects, bacteria and larger organisms communicate with peers by means of pheromones. ${ }^{[1]}$ Communication may result in strategies of cooperation and enables functionalities that go beyond those carried out by individual agents. Compared to traditional telecommunication technologies, molecular communication offers interesting features such as the small (nanoscale) size of molecular transceivers and receivers, the low power consumption required and the ability to operate in biological and physiological environments. ${ }^{[2]}$ Despite its potential to enable future technologies,

[a] B. de Luis, Dr. A. Llopis-Lorente, P. Rincón, Dr. F. Sancenón, Dr. E. Aznar, Dr. J. R. Murguía, Prof. R. Martínez-Máñez Instituto Interuniversitario de Investigación de Reconocimiento Molecular y Desarrollo Tecnológico (IDM), Universitat Politècnica de València, Universitat de València, Camino de Vera s/n, 46022 Valencia, Spain. E-mail: rmaez@qim.upv.es

[b] B. de Luis, Dr. A. Llopis-Lorente, Dr. F. Sancenón, Dr. E. Aznar, Dr. J. R. Murguía, Prof. R. Martínez-Máñez

CIBER de Bioingeniería, Biomateriales y Nanomedicina (CIBERBBN), Spain.

[c] Prof. R. Martínez-Máñez

Unidad Mixta UPV-CIPF de Investigación en Mecanismos de Enfermedades y Nanomedicina, Universitat Politècnica de

València, Centro de Investigación Príncipe Felipe, Valencia, Spain.

[d] Prof. R. Martínez-Máñez

Unidad Mixta de Investigación en Nanomedicina y Sensores,

Universitat Politècnica de València, Instituto de Investigación

Sanitaria La Fe, Valencia, Spain.

[e] Dr. J. Gadea

Instituto de Biología Molecular y Celular de Plantas (IBMCP), Universitat Politècnica de València (UPV)-Consejo Superior de Investigaciones Científicas (CSIC), Valencia, Spain.

[f] Dr. Reynaldo Villalonga

Nanosensors \& Nanomachines Group, Department of Analytical Chemistry, Faculty of Chemistry, Complutense University of Madrid, Madrid, Spain.

Supporting information for this article is given via a link at the end of the document the design of molecular communication systems capable of transmitting information using chemical messengers between different micro/nanoscale entities is not an easy task and remains almost unexplored..$^{[3]}$ An appealing application in this field is to develop communication protocols of living cells with abiotic nanodevices. ${ }^{[4]}$ Biological entities intrinsically react to their environment by means of molecular communication paths decoding the received stimuli and adapting to new conditions. ${ }^{[5]}$ On the other hand, advances in nanoarchitectonics, materials science and synthetic biology can provide the tools to design smart ensembles capable of translating a wide array of (bio)chemical stimuli into encoded messages for the next member of the information chain. ${ }^{[6]}$ Recently, abiotic lipid vesicle-based protocells, microdroplets or fusion protein assemblies capable of translating molecular information from the environment into a response from bacteria have been developed, ${ }^{[7]}$ yet, similar systems using nanoparticles have not been reported.

In communication theory terms, communication is the process in which information is transmitted from one point to another. ${ }^{[8]}$ In the linear model, the simplest communication scheme, the sender converts information into a code and this message is decoded by the receiver on another point. In the interactive model, a more sophisticated strategy, there is a feedback process (Scheme 1A). First, a message is encoded by the sender and transmitted to the decoding receiver. Straightaway, functions reverse and the receiver encodes and transmits a response to the original sender which now acts as receiver. This two-way process facilitates building consortia between different populations to target a common goal.

Here we present, to the best of our knowledge, the first interactive model of communication between living microorganisms (budding yeast Saccharomyces cerevisiae) and abiotic nanodevices (enzyme-controlled Janus Au-mesoporous silica nanoparticles), in which a molecular communication cascade is triggered in response to an environmental stimulus. The expression of green fluorescent protein (GFP) in the cytoplasm of $S$. cerevisiae cells $\mathbf{s}^{[9]}$ is produced as the output signal of the communication system. As illustrated in Scheme 1B, this biunivocal communication starts with the input of sucrose (1), which is detected and transformed by the yeast into glucose and fructose (2). Glucose acts as a chemical messenger that is then transmitted and sensed by glucose oxidase (GOx) on the abiotic nanodevice (3). Transformation of glucose to gluconic acid induces the opening of the gatekeeapers on the mesoporous nanocarrier (4). Subsequently, phleomycin (a second chemical messenger) is released as a feedback message (5) to the microogamism that decodes the information and activates GFP transcription (6) producing a fluorescent signal.

Regarding the design of the chemical communication system, we used budding yeast as an eukaryotic cell model of easy handling and manipulation. ${ }^{[10]}$ Yeast cell wall is mainly composed of polysaccharides, proteins and lipids and avoids the internalization of nanoparticles unless permeability treatments 


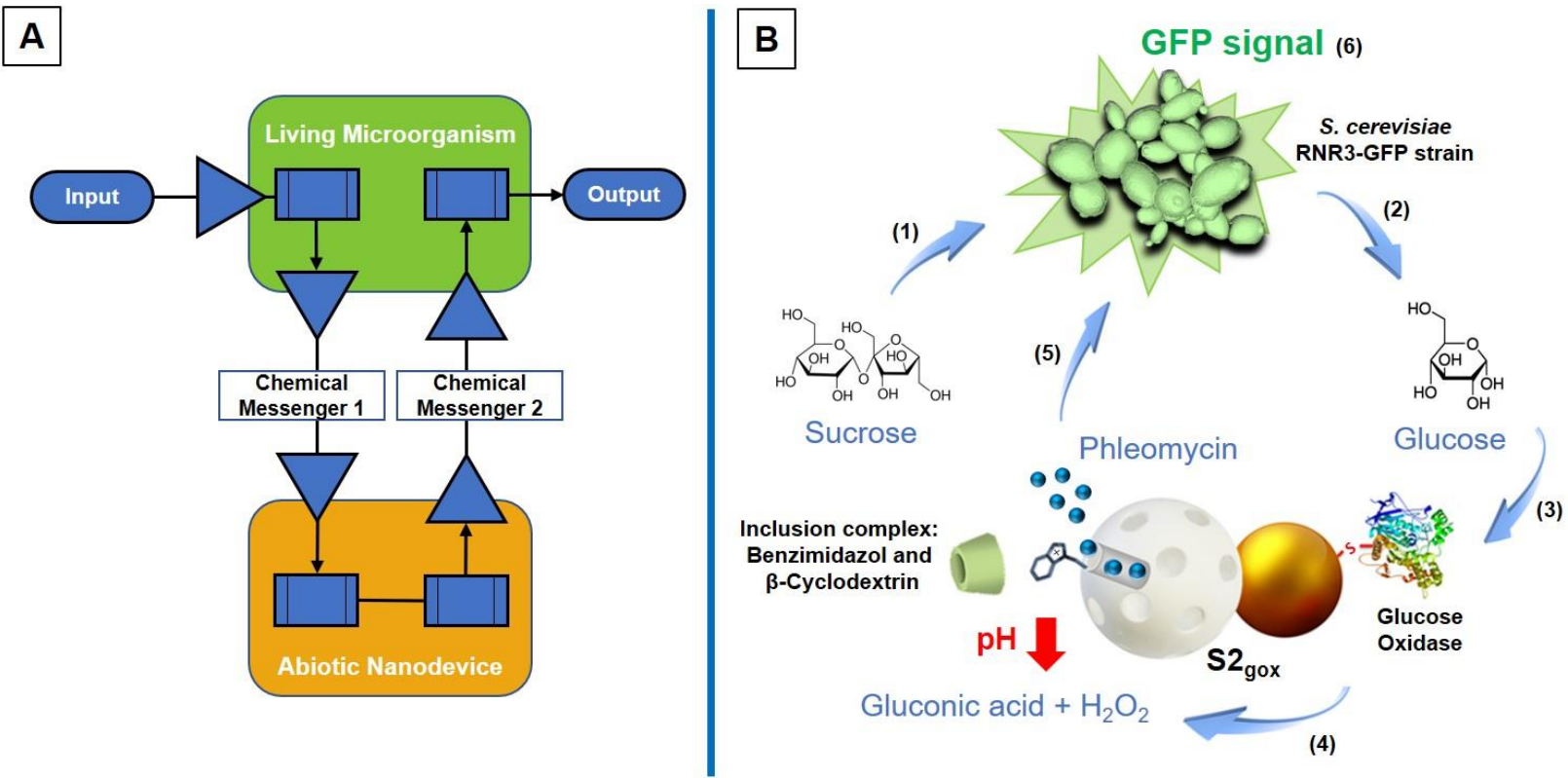

Scheme 1. (A) Representation of the proposed interactive model of communication between living microorganisms and abiotic nanodevices following a flowchart symbology as used in telecommunications. (B) Illustration of the sucrose-responsive interactive system of communication between GFP-expressing S. cerevisiae yeasts and Janus Au-mesoporous silica nanoparticles.

such as electroporation, high temperature incubation and presence of monovalent cations are applied. ${ }^{[11]}$ This fact assures that the interaction process occurs via molecular communication through the medium and not due to nanopartilces internalization. The yeast strain used in our studies expresses GFP under the control of the RNR3 promoter. RNR3 gene transcription is induced upon exposure to DNA-damaging agents. Accordingly, GFP expression is triggered in the selected strain in presence of a genotoxic such as phleomycin. ${ }^{[12]}$ As nanodevice capable of producing a programmed response when a specific stimulus is present, the Janus Au-mesoporous silica architecture is employed as a versatile platform whose double-face nature allows designing systems with a double receiver-sender behaviour. ${ }^{[13]}$ Moreover, although biomimetic protocells have been previously used in communication processes, the use of mesoporous silica scaffolds offers additional appealing properties such as: high thermal and chemical stability, large loading capacity, versatility regarding the nature of cargos and easy synthesis and functionalization with stimuli-responsive gatekeepers and targeting groups.

In our designed systems, the gold particle with immobilized enzymes operates as a control unit processing the chemical information received from the environment and emitting a response which regulates the nanovalve uncapping on the mesoporous face. In particular, the pH-responsive supramolecular nanovalve we have used consists of an inclusion complex between a benzimidazole moiety and $\beta$-cyclodextrin. Local gluconic acid produced by GOx causes the protonation of benzimidazole group $\left(\mathrm{pK}_{\mathrm{a}}=5.55\right)^{[14]}$ and therefore the dethreading of the complex leading to the delivery of the entrapped cargo. Notwithstanding, a variety of molecular and supramolecular gatekeepers have been developed in recent years which can be used for developing future communication systems. ${ }^{[15]}$
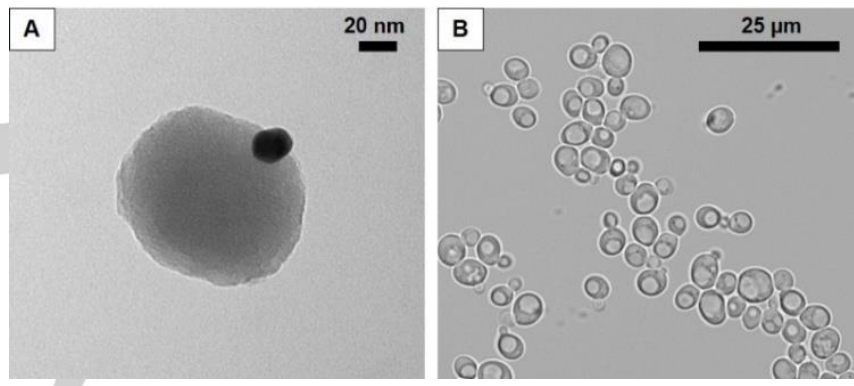

Figure 1. Images of the nanodevice and microorganism employed in the communication network. (A) Transmission electron microscopy (TEM) image of a Janus Au-mesoporous nanoparticle and (B) transmitted light image of Saccharomyces cerevisiae budding yeasts obtained by confocal microscopy.

In a first step, after nanoparticle preparation and characterization by different techniques (see Supporting Information), we tested the ability of the nanodevice to recognize glucose and release the cargo with the dye $\left(\left[\mathrm{Ru}(\mathrm{bpy})_{3}\right] \mathrm{Cl}_{2}\right)$-loaded nanoparticles ( $\left.\mathrm{S} 1_{\text {gox }}\right)$. With this aim, we brought solid $\mathrm{S} 1_{\text {gox }}$ to a concentration of $1 \mathrm{mg} \cdot \mathrm{mL}^{-1}$ in aqueous solution at $\mathrm{pH} 7.5$ and monitored cargo release in the absence and presence of the input (glucose $1 \mathrm{mM}$ ). Aliquots were taken at scheduled times, centrifuged to remove nanoparticles and the fluorescence of the supernatants was measured (emission band of $\left[\mathrm{Ru}(\mathrm{bpy})_{3}\right] \mathrm{Cl}_{2}$ at $595 \mathrm{~nm}, \lambda_{\mathrm{exc}}=453 \mathrm{~nm}$ ). The delivery studies showed negligible dye release in absence of glucose whereas the presence of the input led to opening of pores and the subsequent remarkable release of the fluorescent dye (Figure SI-8). As explained above, this is due to the activity of GOx which oxidized glucose to gluconic acid contributing to the protonation of benzimidazole. A selective sensing-actuating behaviour is a highly desired characteristic for a component of a communication network. Thus, we confirmed the specificity of the nanodevice by carrying out delivery experiments with $\mathrm{S} 1_{\text {gox }}$ in the presence of different monosaccharides and disaccharides. In these studies, no 
noticeable $\left[\mathrm{Ru}(\mathrm{bpy})_{3}\right] \mathrm{Cl}_{2}$ delivery was observed after 3 hours as these species are not recognized by the GOx enzyme on the gold unit (Figure $\mathrm{SI}-10$ ).

Next, we evaluated the linear yeast-nanodevice communication pathway as an initial key step in order to build the interactive model of communication. To this end, we carried out delivery studies combining both elements, i.e. yeast and the mesoporous nanocarriers $S 1_{\text {gox }}$ in solution. For these experiments, dye-loaded solid $\mathrm{S} 1_{\text {gox }}$ and yeast were brought to a final concentration of $1 \mathrm{mg} \cdot \mathrm{mL}^{-1}$ and $1.5 \cdot 10^{8}$ cells $\cdot \mathrm{mL}^{-1}$, respectively, in water at $\mathrm{pH} 7.5$. Then, dye release was monitored by fluorescence spectroscopy in the absence and presence of the input (sucrose 2\%). As shown in Figure 2, the results validate the establishment of a linear communication pathway between the microorganism and the nanodevice in the presence of sucrose (blue curve, $74.8 \%$ release efficiency). In contrast, no dye release was triggered in the absence of sucrose (red curve) nor in the presence of sucrose and absence of yeast (black curve). This sucrose-recognition capability of the communication system is ascribed to the abundance of invertase enzyme in the periplasmic space and cell walls of Saccharomyces cerevisiae ${ }^{[16]}$ (catalysing the hydrolysis of sucrose into fructose and glucose) and the subsequent connection with the glucose-responsive nanocarrier. Moreover, we also demonstrated that the $\left[\mathrm{Ru}(\mathrm{bpy})_{3}\right] \mathrm{Cl}_{2}$ released in these experiments did not induce significant toxicity in yeast cells (Figure SI-11).

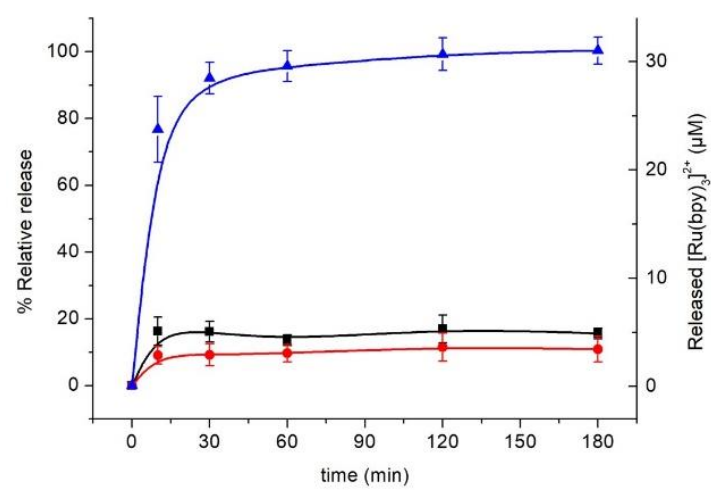

Figure 2. Validation of the sucrose-responsive linear communication pathway between the microorganism and the enzyme-controlled nanodevice, as determined by dye delivery studies. Kinetics of $\left[\mathrm{Ru}(\mathrm{bpy})_{3}\right] \mathrm{Cl}_{2}$ release in aqueous solution at $\mathrm{pH} 7.5$ that contained $\mathrm{S} 1_{\text {gox }}$ and yeasts in the absence (red curve) and presence (blue curve) of sucrose (2\%). A negative control (only sucrose and nanoparticles) was also performed (black curve). Error bars correspond to the s.d. from three independent experiments.

On a further step towards the construction of the interactive model of communication, we prepared nanoparticles loaded with the genotoxic phleomycin which was expected to act as a feedback messenger in order to close the communication loop. Delivery kinetics (Figure SI-9) from the phleomycin-loaded nanodevice $\left(\mathrm{S}_{\text {gox }}\right.$ ) were determined by measuring phleomycin absorbance at $300 \mathrm{~nm}$ in solution in the presence and absence of glucose $\left(1 \%\right.$, nanoparticle conc. $\left.=2.5 \mathrm{mg} \cdot \mathrm{mL}^{-1}\right)$. After 3 hours, the supernatants were added to fresh $S$. cerevisiae cultures (inoculated in yeast extract peptone dextrose (YPD) medium) and the mixtures were shaken at $28^{\circ} \mathrm{C}$ for another two hours. Then, the GFP signal expression was evaluated by confocal microscopy. Furthermore, positive and negative control experiments were carried out by adding or not free phleomycin to yeast cultures and incubating for two hours. A similar fluorescent response was obtained from yeast treated with free phleomycin and treated with phleomycin released from $\mathrm{S}_{\text {gox }}$ in the presence of glucose (Figure SI-12). These results confirmed the potential of the nanodevice $S 2_{\text {gox }}$ to induce GFP expression in yeast upon glucose-inducted delivery.
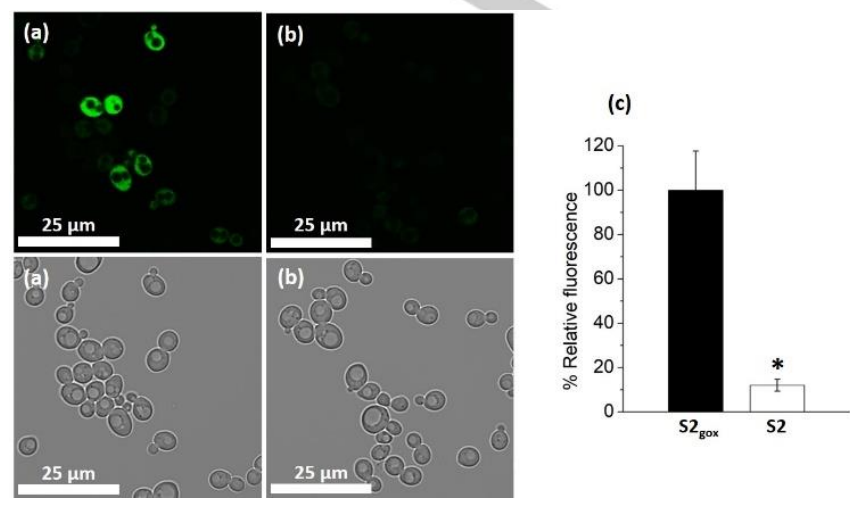

Figure 3. GFP signal induction in $S$. Cerevisiae cells examined by confocal microscopy after 2 hours for (a) incubation with $\mathrm{S} 2_{\text {gox }}$ and sucrose $1 \%$ and $(\mathrm{b})$ incubation with $\mathrm{S} 2$ and sucrose $1 \%$. Above: fluorescence images, below: transmission images. Additional images are provided in the Supporting Information (Figure SI-13). (c) Normalized quantification of the GFP-associated fluorescence intensity for the two different treatments. Several fields of each condition in three independent experiments were analyzed obtaining similar results. Data represent the mean \pm s.e.m. of three independent experiments $\left({ }^{*} p\right.$ $<0.01)$.

Encouraged by these findings, we addressed the complete interactive communication model in which the microorganism and the nanodevice $S 2_{\text {gox }}$ were expected to exchange chemical messengers through an aqueous medium. Yeast microorganisms were inoculated in fresh YPD and incubated until reaching midlog exponential phase. Then, yeasts were washed three times (by centrifugation) and resuspended in YPD medium without glucose. For the final experiments, aqueous solutions at $\mathrm{pH} 7.5$ containing the nanodevice $\mathrm{S} 2_{\text {gox }}\left(10 \mu \mathrm{g} \cdot \mathrm{mL}^{-1}\right.$ of final concentration) were mixed with yeast suspensions. Then, $1 \%$ of sucrose (input) was added as energy source necessary for gene expression in yeasts ${ }^{[17]}$ and initiator of the communication network. Same experiments were carried out with nanoparticles S2 (lacking the GOx enzyme) as a control. Confocal microscope images showed that the communication system displayed a clear GFP-associated fluorescence when $\mathrm{S}_{\text {gox }}$ was present (Figure $3 \mathrm{a}$ and $\mathrm{SI}-13 \mathrm{a}$ ). On the contrary, the fluorescent signal was negligible when S2 (lacking GOx) was used (Figure 3b and SI-13b). Quantification of the GFP-associated fluorescence intensity by analysis of the confocal images (from three independent experiments), revealed a marked 8-fold enhancement in cell emission for the complete yeast-S2 $2_{\text {gox }}$ communication system (Figure 3c). As additional control experiments, we prepared nanodevice $\mathrm{S}_{\text {gox }}$ (lacking the cargo) and nanodevice S3 (lacking both the cargo and GOx enzyme). As expected, no communication and therefore no GFP expression was observed when these nanoparticles were used (Figure SI-14). Altogether, confocal imaging experiments demonstrated that only in the presence of all components of the communication network, the GFP output signal was achieved. 
When the GOx enzyme or the cargo in the nanoparticles were missing, the communication channel was disrupted and information was lost (Table SI-4). Overall, the behaviour of this hybrid interactive communication system between yeasts and nanoparticles can be summarized in a Boolean logical table (Table 1) and corresponds to a 4-input 3-level AND logic gate (Scheme 2).

Eventually, it was demonstrated by cell viability assays based on CFUs counting after $48 \mathrm{~h}$ that the unloaded nanodevice $\left(\mathrm{S} 3_{\text {gox }}\right)$ was not toxic for the Saccharomyces cerevisiae yeast (Figure SI-15). In contrast, S2 ${ }_{\text {gox }}$ in the presence of sucrose induced a significant reduction in cell viability to only $30 \%$. This is due to the release of phleomycin which acts a genotoxic producing activation of GFP expression and leading to cell death. Therefore, these results correlate well with the confocal imaging.

Table 1. Summary of the behaviour of the communication system in Boolean logic notation (truth table). Presence of a component is summarized as 1 and absence with 0 . In presence of the stimulus (1), the output signal (1) is only produced when all components are present. The output signal is considered 0 when the normalized quantification of the GFP-associated fluorescence intensity is less than $20 \%$ of that found for the complete systems, i.e. Input 1, Yeast 1, Enzyme 1 and Cargo 1.

\begin{tabular}{|c|c|c|c|c|}
\hline Input ${ }^{[a]}$ & Yeast $^{[\mathrm{b}]}$ & Enzyme $^{[c]}$ & Cargo $^{[\mathrm{d}]}$ & Output $^{[e]}$ \\
\hline 0 & 1 & 1 & 1 & 0 \\
\hline 1 & 0 & 1 & 1 & 0 \\
\hline 1 & 1 & 0 & 1 & 0 \\
\hline 1 & 1 & 1 & 0 & 0 \\
\hline 0 & 0 & 1 & 1 & 0 \\
\hline 0 & 1 & 0 & 1 & 0 \\
\hline 0 & 1 & 1 & 0 & 0 \\
\hline 1 & 0 & 0 & 1 & 0 \\
\hline 1 & 0 & 1 & 0 & 0 \\
\hline 1 & 1 & 0 & 0 & 0 \\
\hline 1 & 0 & 0 & 0 & 0 \\
\hline 0 & 1 & 0 & 0 & 0 \\
\hline 0 & 0 & 1 & 0 & 0 \\
\hline 0 & 0 & 0 & 1 & 0 \\
\hline 0 & 0 & 0 & 0 & 0 \\
\hline 1 & 1 & 1 & 1 & 1 \\
\hline
\end{tabular}

[a] Sucrose $1 \%$ [b] $S$. cerevisiae budding yeast [c] glucose oxidase in capped nanoparticles [d] phleomycin as cargo in capped nanoparticles [e] GFP fluorescent signal.

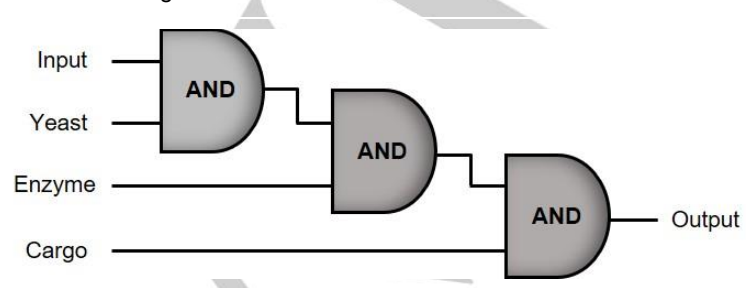

Scheme 2. 4-input 3-level AND logic gate corresponding to the logic Table 1 describing the behaviour of the communication system.

In conclusion, we have developed for the first time an interactive model of communication between abiotic nanodevices and living microorganisms in which communication is triggered by environmental agents. The information obtained by the emisor (yeast cell) from the surroundings (sucrose as stimulus) is processed and transmitted to the receiver (nanoparticles) which in turn is capable of decoding the information and transmitting a response back to the original sender. This communication network based on a biunivocal scheme employs enzymatic reactions and chemical messengers to enable an action which could not be achieved in isolation (induction of GFP expression by yeasts). In spite of being a proof of concept, these findings demonstrate how abiotic nanodevices and living microorganisms can be connected using molecular communication yielding multicomponent systems with a collective synergic behaviour. In addition, considering the large variety of molecules produced and secreted by living microorganisms which can be processed by enzymatic receptors as well as the numerous different species potentially susceptible to be encapsulated in mesoporous silica nanoparticles, we believe that the proposed communication network represents a general versatile paradigm which can help laying the foundations of future research toward the development of novel technologies based on communication at the nanoscale. We also envision that the combination of different enzymes integrated on the same nanodevice could mimic the biological cascade necessary to synthesize in-situ bioactive or functional molecules from precursors present in the environment, produced by organisms or released from the mesoporous support. Such insitu produced bioactive or functional molecules could be implemented into the information network further increasing the scope of the proposed communication paradigm. Moreover, we also believe that the idea of tailoring synthetic nanodevices ${ }^{[18]}$ and communicating them with cells can have impact in the design of new sensors, new strategies for diagnosis and personalized therapeutics. ${ }^{[19]}$

\section{Acknowledgements}

B. de Luis is grateful to the Spanish Government for her FPU PhD fellowship. The authors wish to thank the Spanish Government (projects RTI2018-100910-B-C41 (MCUI/AEI/FEDER, UE) and CTQ2017-87954-P), the Generalitat Valenciana (project PROMETEO2018/024), the Comunidad de Madrid (Project IND2017/BMD-7642) and CIBER-BBN (NANOCOM project) for support.

Keywords: molecular communication - abiotic nanodevices • living microorganisms $\bullet$ nanonetworks $\bullet$ mesoporous materials

[1] a) B. Alberts, A. Johnson, J. Lewis, D. Morgan, M. Raff, K. Roberts, P. Walter in Molecular Biology of the Cell, 6th Ed., Garland Science, 2015; b) C. M. Waters, B. L. Bassler, Annu. Rev. Cell Dev. Biol. 2005, 21, 319346; c) J. Fleischer, J. Krieger. Front. Cell Neurosci. 2018, 12, 425; d) S. Nahavandi, S.-Y. Tang, S. Baratchi, R. Soffe, S. Nahavandi, K. Kalantarzadeh, A. Mitchell, K. Khoshmanesh, Small 2014, 10, 4810-4826.

[2] a) I. F. Akyildiz, F. Brunetti, C. Blánzquez, Comp. Netw. 2008, 52, 2260 2279; b) G. Li-Destri, L. Fichera, A. Zammataro, G. T. Sfrazzetto, N. Tuccitto, Nanoscale 2019, doi: 10.1039/C9NR04461B; c) N. Tuccitto, G. LiDestri, G. M. L. Messina, G. Marletta, Phys. Chem. Chem. Phys. 2018, 20, 30312-30320. 
[3] A. Llopis-Lorente, P. Díez, A. Sánchez, M. D. Marcos, F. Sancenón, P. Martínez-Ruiz, R. Villalonga, R. Martínez-Máñez, Nano Today 2018, 18 8-11.

[4] a) J. L. Marzo, J. M. Jornet, M. Pierobon, Curr. Drug Targets 2019, 20 800-807; b) E. J. Kwon, J. H. Lo, S. N. Bhatia, PNAS 2015, 112, 1446014466; c) Y. Benenson, Nat. Rev. Genet. 2012, 13, 455-468.

[5] a) D. Barcena Menendez, V. R. Senthivel, M. Isalan, Curr. Opin Biotechnol. 2015, 31, 101-107; b) D. Malak, O. B. Akan, Nano. Commun Netw. 2012, 3, 19-35.

[6] a) M. Komiyama, K. Yoshimoto, M. Sisido, K. Ariga, Bull. Chem. Soc Jpn. 2017, 90, 967-1004; b) P. Stano, G. Rampioni, P. Carrara, L. Damiano, L. Leoni, P. L. Luisi BioSystems 2012, 109, 24-34; c) A. LlopisLorente, P. Díez, A. Sánchez, M. D. Marcos, F. Sancenón, P. MartínezRuiz, R. Villalonga, R. Martínez-Máñez, Nat. Comm. 2017, 8, 15511; d) C. Giménez, E. Climent, E. Aznar, R. Martínez-Máñez, F. Sancenón, M. D. Marcos, P. Amorós, K. Rurack, Angew. Chem. Int. Ed. 2014, 53 , 12629-12633; e) R. Lentini, N. Y. Martín, S. S. Mansy, Curr. Opin. Chem. Biol. 2016, 34, 53-61; f) Y. Ding, L. E. Contreras-Llano, E. Morris, M. Mao C. Tan, ACS Appl. Mater. Interfaces 2018, 10, 30137-30146; g) K. Ariga, X. Jia, J. Song, C.-T. Hsieh, S.-h. Hsu, ChemNanoMat. 2019, 5, 692;

[7] a) R. Lentini, S. Perez Santero, F. Chizzolini, D. Cecchi, J. Fontana, M. Marchioretto, C. Del Bianco, J. L. Terrell, A. C. Spencer, L. Martini, M. Forlin, M. Assfalg, M. Dalla Serra, W. E. Bentley, S. S. Mansy, Nat Comm. 2014, 5, 4012; b) R. Lentini, N. Y. Martín, M. Forlin, L. Belmonte, J. Fontana, M. Cornella, L. Martini, S. Tamburini, W. E. Bentley, O Jousson, S. S. Mansy, ACS Cent. Sci. 2017, 3, 117-123; c) G. Rampioni, F. D'Angelo, M. Messina, A. Zennaro, Y. Kuruma, D. Tofani, L. Leoni, P. Stano, Chem. Comm. 2018, 54, 2090-2093; d) M. Schwarz-Schilling, L. Aufinger, A. Mückl, F. C. Simmel, Integr. Biol. 2016, 8, 564-570; e) R. Fernandes, V. Roy, H.-C. Wu, W. E. Bentley, Nat. Nanotechnol. 2010, 5 213-217; f) A. Gupta, J. L. Terrell, R. Fernandes, M. B. Dowling, G. F. Payne, S. R. Raghavan, W. E. Bentley, Biotechnol Bioeng. 2012, 110, 552-562.

[8] J. S. Chitode in Communication Theory, Technical Publications, Pune, 2010
[9] W.-K. Huh, J. V. Falvo, L. C. Gerke, A. S. Carroll, R. W. Howson, J. S Weissman, E. K. O'Shea, Nature 2003, 425, 686-691.

[10] S. M. Burgess, T. Powers, J. C. Mell, eLS 2017, DOI 10.1002/9780470015902.a0000821.pub2.

[11] a) N. Mas, I. Galiana, S. Hurtado, L. Mondragón, A. Bernardos, F. Sancenón, M. D. Marcos, P. Amorós, N. Abril-Utrillas, R. MartínezMáñez, J. R. Murguía, Int. J. Nanomed. 2014, 9, 2597-2606; b) A. Agostini, L. Mondragón, A. Bernardos, R. Martínez-Máñez, M. D. Marcos, F. Sancenón, J. Soto, A. Costero, C. Manguan-García, R. Perona, M. Moreno-Torres, R. Aparicio-Sanchis, J. R. Murguía, Angew. Chem. Int Ed. 2012, 51, 10556-10560.

[12] Y. Endo-lchikawa, H. Kohno, R. Tokunagat, S. Taketani, Biochem Pharmacol. 1995, 50, 10, 1695-1699.

[13] R. Villalonga, P. Díez, A. Sánchez, E. Aznar, R. Martínez-Máñez, J. M. Pingarrón, Chem. Eur. J. 2013, 19, 7889-7894.

[14] G. Jerez, G. Kaufman, M. Prystai, S. Schenkeveld, K. K. Donkor, J. Sep. Sci. 2009, 32, 1087-1095.

[15] a) E. Aznar, M. Oroval, L. Pascual, J. R. Murguía, R. Martínez-Máñez, F. Sancenón, Chem. Rev. 2016, 116, 561-718.

[16] P. M. Gomes Barbosa, T. Pereira de Morais, C. A. de Andrade Silva, F R. da Silva Santos, N. F. Lisbo Garcia, G. G. Fonseca, R. S. Ribeiro Leite, M. F. da Paz, Prep. Biochem. Biotechnol. 2018, 48, 506-513.

[17] M. P. Ashe, S. K. De Long, A. B. Sachs, Mol. Biol. Cell 2000, 11, 833848.

[18] a) K. Ariga, D. T. Leong, T. Mori, Adv. Funct. Mater. 2018, 28, 1702905; b) K. AC. Xu, S. Hu, X. Chen, Mater. Today 2016, 19, 516-532.

[19] a) S. Hauert, S. N. Bhatia, Trends Biotechnol. 2014, 32, 448-455; b) I. F. Akyildiz, M. Pierobon, S. Balasubramaniam, Proc. IEEE 2019, 107, 858 865 ; c) S. G. Hays, W. G Patrick, M. Ziesack, N. Oxman, P. A. Silver, Curr. Opin. Biotechnol. 2015, 36, 40-49; d) F. Peng, Y. Tu, D. A. Wilson, Chem. Soc. Rev. 2017, 46, 5289-5310; e) E. Morris, M. Chavez and C Tan, Curr. Opin. Biotechnol. 2016, 39, 97-104; f) F. Peng, Y. Tu, J. C. M van Hest, D. A. Wilson, Angew. Chem. 2015, 127, 11828-11831. 
Entry for the Table of Contents (Please choose one layout)

Layout 1:

\section{COMMUNICATION}

As a proof-of-concept, we report herein an interactive paradigm of communication between yeasts and enzyme-controlled Janus Aumesoporous silica nanoparticles. The fluorescent signal from the microorganism (corresponding to the production of green fluorescent protein) is governed by the biunivocal communication with the nanodevice via enzymatic reactions and the exchange of chemical messengers.

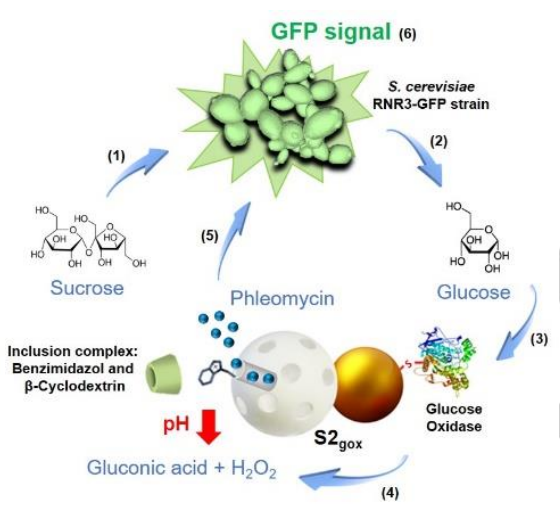

Beatriz de Luis, Antoni Llopis-Lorente, Paola Rincón, José Gadea, Félix Sancenón, Elena Aznar, Reynaldo Villalonga, José Ramón Murguía and Ramón Martínez-Máñez

Page No. - Page No.

An Interactive Model of Communication between Abiotic Nanodevices and Living Microorganisms 